\title{
Tensile Comparison of Polymer Specimens Produced with Different Processes
}

\section{Dr. Wei Dai Vian, Purdue University, West Lafayette}

Dr. Wei Vian is an assistant professor in practice in the program of Mechanical Engineering Technology at Purdue University Statewide Kokomo campus. She got her Ph.D from Purdue Polytechnic, Purdue University, West Lafayette. She got her bachelor and master degree both from Eastern Michigan University. Her recent research interests include grain refinement of aluminum alloys, metal casting design, and innovation in engineering technology education.

\section{Prof. Nancy L. Denton P.E., Purdue University, West Lafayette}

Nancy L. Denton, PE, CVA3, is a professor in Purdue University's School of Engineering Technology, where she serves as associate head for MET. She is on the Vibration Institute's Board of Directors, chairing the Academic Committee and serving on the Certification Scheme Committee. She is a Fellow of ASEE and a member of ASME. 


\title{
Tensile Comparison of Polymer Specimens Produced by Different Processes
}

\begin{abstract}
The tensile test identifies key mechanical properties of materials, making it a common element in many materials science laboratory classes. In the sophomore-level mechanics course for the mechanical engineering technology (MET) degree program and the introductory materials and processes courses for MET and manufacturing engineering technology (MFET) students at campuses of XXXXX University, tensile testing of dogbone specimens made by a single manufacturing process has been in place for many years. As is, this testing provides an effective means for lower division college students to understand how mechanical properties such as Young's modulus and ultimate tensile strength are determined. However, the variability in apparent and actual property values that comes from differences in manufacturing processes and dimensional tolerances leads to student confusion and lack of confidence in their experimental results. To help lower division college students understand how these factors affect experimental results, the tensile testing experiment was expanded to incorporate thermoplastic specimens made by three distinctly different processes. Student roles included manufacture of the specimens, measurement of necessary dimensions, conducting the tensile tests, generating stressstrain curves for each specimen, and determining the corresponding Young's modulus and ultimate tensile strength values. Reflection on the effects of process and geometric variation on their property results became a new report requirement. This article will present the complete laboratory experience, results, and initial student response to this enhanced tensile testing experiment.
\end{abstract}

Keywords: Tensile test, Tensile strength, Specimen preparation, Specimen manufacture 


\section{Background}

The stiffness and strength of a material are primary concerns for engineers. These properties typically come from the uniaxial tensile test, one of the most common static materials tests ${ }^{1}$. College students in XXXXX University's MET program perform tensile testing of different materials in several materials and mechanics courses. For polymer materials, tensile testing normally follows the requirements of ASTM D638 ${ }^{2}$. Students complete their laboratory testing and process their tensile force and extension data to generate a stress-strain plot and identify corresponding properties. In their discussion and analysis, one element often ignored by students is the quality of the specimen provided for testing. Preparation of a specimen for testing actually is an essential part of the testing

process because the standard specimens ensure meaningful and reproducible results. ${ }^{1}$ To help improve students' critical thinking, hands-on experience, and potential research interest, an enhanced tensile testing laboratory project was developed that accounts for specimen condition and variability.

MET students at two campuses of XXXXX University participated in this enhanced polymer tensile testing laboratory project. Campus $\mathrm{A}$ is a commuter campus with a balanced population mix of traditional and non-traditional students and typical engineering technology class sizes of 10-20. Campus B is a large residential campus populated by traditional students, transfer students, and a handful of non-traditional students. At Campus A, the participants are sophomore students in the basic strength of materials course. The student from Campus B who contributed to this project was a freshman seeking an initial undergraduate research experience while enrolled in an introductory materials and processes course.

\section{Introduction}

From the students' perspective, the objectives of the polymer tensile testing laboratory project are to explore the tensile mechanical properties, to learn the testing technique, and to interpret the ASTM standard test method. Resistance to tensile loading is an important material characteristic for plastics. In fact, this property is often the main factor that limits use of plastics in engineering applications that will be exposed to a range of temperatures ${ }^{3}$. Like other materials, plastics are often specified in a design for their ability to withstand loading in tension. ASTM D638 - Standard Test Method for Tensile Properties of Plastics - is one of the most widely accepted methods for evaluating tensile properties, and is generally followed in this laboratory project. The test results provide an engineering stress-strain curve for material properties such as modulus of elasticity, tensile strength, and elongation. 
Previous tensile testing by engineering technology students at XXXXX University used pre-made specimens. Students used these specimens to obtain load and deformation data, generate stress-strain curves, identify Young's modulus and tensile strength, then compared the resulting property values with their published counterparts. Unfortunately, the resulting lab reports reflected minimal student cognition regarding the importance of specimen preparation and quality, which directly affects the testing results.

To encourage the students' learning interests, develop their scientific research skills, and enhance lecture knowledge, a new tensile testing laboratory project has been designed and implemented for MET at Campus A. Elements of the project were duplicated at Campus B by a freshman undergraduate researcher for comparison.

\section{Design of the Tensile Laboratory Project}

This laboratory project combines manufacturing with tensile testing. Students design the tensile specimen CAD model, manufacture the specimens, conduct their testing, and complete their data analysis. For the project, specimen materials are several common thermoplastics, including polystyrene (PS), high-density polystyrene (HIPS), low-density polyethylene (LDPE), acrylonitrile butadiene styrene (ABS), and polylactic acid (PLA). These thermoplastics have relatively low strength and can be shaped (molded) at relatively low temperatures. Three manufacturing processes were applied in this project: injection molding, 3-D printing, and CNC machining of rolled sheet. Injection molding is one of the most common traditional methods used to process thermoplastics. The material is melted, forced into a mold cavity, and cooled. A much newer process that has become increasingly popular in producing polymer parts, continuous filament deposition 3-D printing allowed students to utilize their design skills. Five different 3-D printers were applied in this project, introducing another layer of variability. CNC machining of commercial polymer sheets (cut-to-size sheet) constituted the third process. The geometry and dimensions of the specimen in all three processes were consistent with ASTM standard D638 specifications. Specimens of each material were produced by at least two of the three manufacturing methods. All specimens were tested for tensile strength characteristics on a universal tensile testing machine equipped with computer software to collect load and deformation data and generate the stress-strain curves from student-entered cross-sectional dimensions and gauge length. A manual length measurement followed the tensile test to determine elongation. Finally, the data and results were organized, calculated, plotted, and analyzed.

The results of the comparison of like material specimens made from different manufacturing processes are presented in this paper from the students' discussion and conclusion. The improvement and modification of each process toward future lab project 
is also included. Through this enhanced laboratory project, students acquired experimental research experience by working through the hands-on design, processing and testing phases of the project, making assumptions and estimations before knowing their experimental results, analyzing the data of testing, and recommending future improvements.

\section{Methodology}

This enhanced laboratory is a group project originally designed for students of Strength of Materials at Campus A. Each group had four members. All the students were in their sophomore year who had already taken entry-level manufacturing and materials courses. The freshman student at Campus B was taking an introductory manufacturing and material class which focused on polymers. Working individually, this student focused on the 3-D printing process and strength testing. The total time spent on this project, which was from introducing the project to submitting the report, was approximately one month. Students used the regular laboratory session (once per week, two hours each time) to complete the work in groups. Assessment elements included a progress list, group peer evaluation form, and final written report. A post-project survey (see appendix B for complete survey questions) served as the concluding project requirement and assessment tool.

\section{$\underline{\text { Limitations }}$}

This was the first implementation of this laboratory project. Although students knew fundamentally how to produce injection molded parts and perform tensile tests from previous lab experiences, they still encountered difficulties during the project. For example, it was hard to set the injection molding process temperatures high enough to melt the ABS pellets and ensure complete fill of the mold cavity. Similarly, because of the relatively high ductility of these thermoplastics, final length measurements were not very precise. The schedule of the project was affected and some planned work was not completed, such as generating actual stress-strain curves by the sophomores. The freshman student researcher continued the work to see effects of actual cross-sectional dimensions on apparent tensile strength

The 3-D printers printed specimens with polymer filament, the injection molding process used polymer pellets, and the cut-to-size CNC-machining occurred for pre-purchased polymer sheets. Each form of the material theoretically had the same chemistry but showed differences in material properties. Additives such as colorants may be the source of these differences in performance of like materials, or the differences could be caused by the manufacturing processes themselves. In the comparison of like material specimens, 
the potential for error caused by raw material differences was assumed to be negligible since the coloring method of the material is unknown. ${ }^{7}$

\section{Manufacturing Processing}

Three different manufacturing processes are applied to make the same size specimens in this project: injection molding, 3-D printing, and CNC machining. The specimen dimensions are from ASTM D638 - Standard Test Method for Tensile Properties of Plastics $^{2}$, as shown in Figure 1. Based on the standard tensile specimen dimensions as shown in Table 1, an aluminum mold was CNC-machined for the injection molding process and appears in Figure 2. PS, LDPE, and ABS pellets, respectively, were poured into the barrel of the molder, heated, and injected into the dogbone-shaped mold cavity through a small sprue (tube opening). The corresponding temperature settings for processing these three materials were $230{ }^{\circ} \mathrm{C}$ (ABS), $110{ }^{\circ} \mathrm{C}(\mathrm{LDPE})$, and $210^{\circ} \mathrm{C}$ (PS). Twelve to fifteen specimens of each polymer were injection molded by the students. The specimens were taken out of the mold and any excess material (flash) from the sprue connection and from between the mold halves was removed, then the specimens were inspected. Common defects were brownish spots (over-burned), uneven surfaces, and missing corners (not enough material). Ten samples with limited defects were selected for testing. Each type of specimen has a different shade of colorant so students could not mix up the samples (see Table 2).

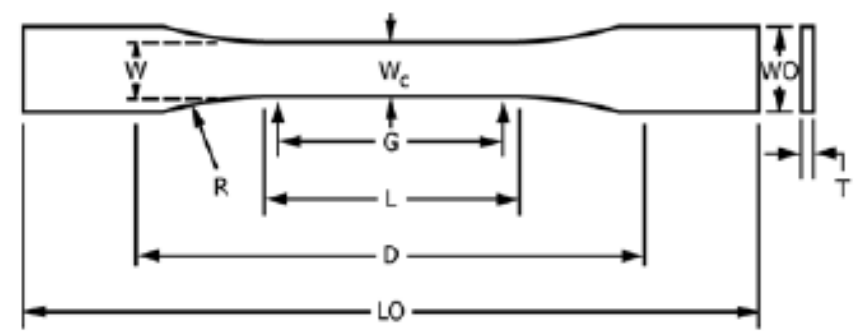

Figure 1 Specimen dimensions (ASTM D638-14²)

Table 1 - ASTM D638-14 Standard Specimen Dimensions ${ }^{2}$

\begin{tabular}{|l|l|}
\hline Type I Specimen & Dimensions: mm (in) \\
\hline T-Thickness & $3(0.13)$ \\
\hline W-Width of narrow section & $13(0.50)$ \\
\hline L-Length of narrow section & $57(2.25)$ \\
\hline WO-Width overall, min & $19(0.75)$ \\
\hline LO-Length overall, min & $165(6.5)$ \\
\hline G-Gage Length & $50(2.00)$ \\
\hline D-Distance between grips & $115(4.5)$ \\
\hline R-Radius of fillet & $76(3.0)$ \\
\hline
\end{tabular}



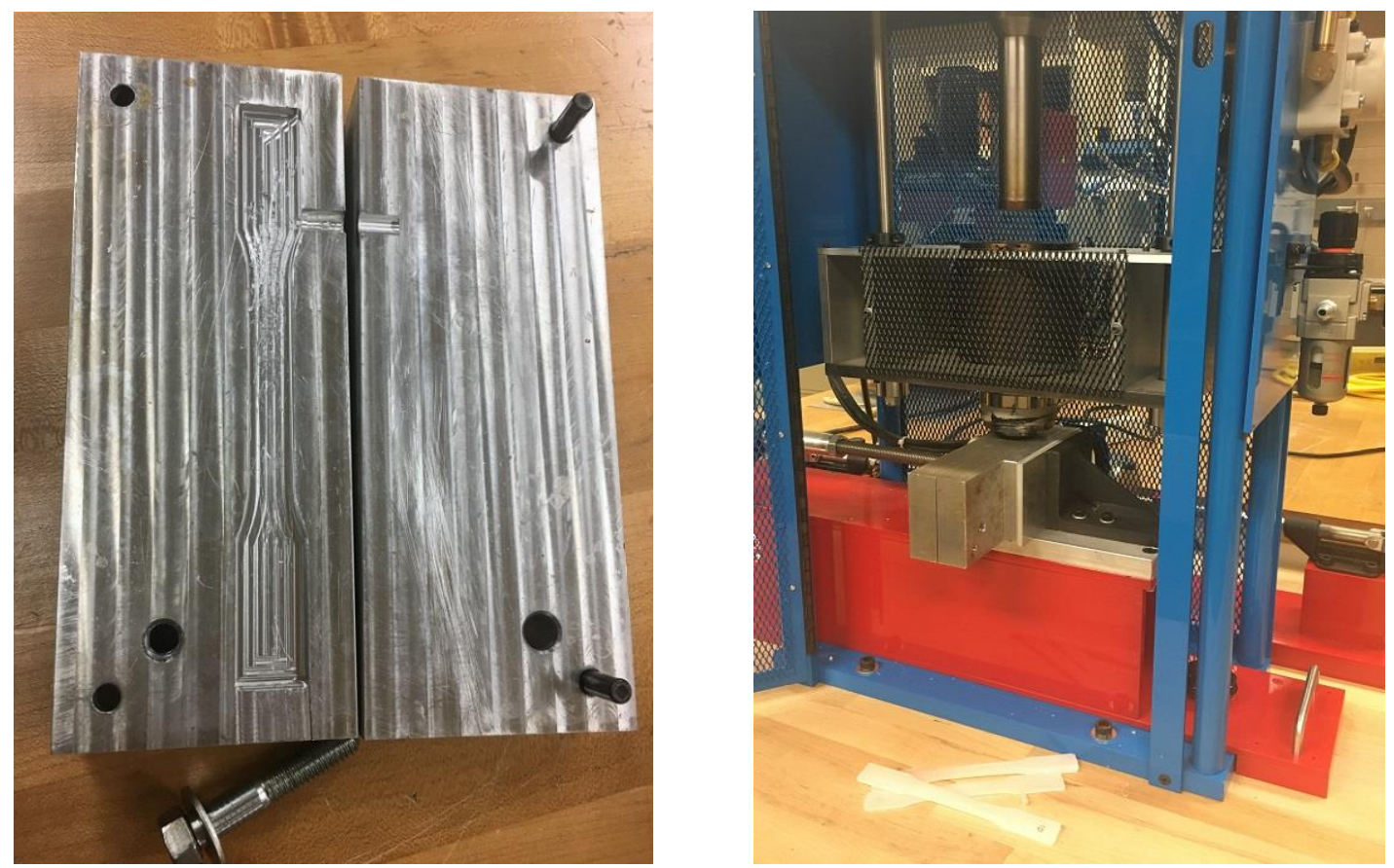

Figure 2 Aluminum Mold for Injection Molding, open (left) and in use (right)

Table 2 - Specimens Types and Colors

\begin{tabular}{|l|l|l|l|}
\hline Polymer & Injection Molding & 3-D Printing & Cut-to-Size Sheet \\
\hline PLA & -- & Gold & -- \\
\hline ABS & Cream & Silver gray & Beige \\
\hline HIPS & -- & Yellow & -- \\
\hline LDPE & White & -- & Clear \\
\hline PS & Clear & -- & White \\
\hline
\end{tabular}

At the same time, a 3-D model of the specimen was created in the computer aided software AutoCAD and saved in the format of standard tessellation language (.STL file) for printing. Five 3-D printers from two campuses used ABS, PLA, and HIPS filaments to complete the work in a week (see table 3 and appendix A for printer information). For each material, approximately 20 specimens were printed at Campus A. For Campus B, five or six specimens per material were printed. The $1.75 \mathrm{~mm}$ and $3 \mathrm{~mm}$ polymer filament for 3-D printing was purchased online, with specifications listed in table 4. 3-D printed specimens have somewhat rough surfaces and they were not as dense as injection molded or cut-to-size parts based on unit weight.

Table 3 - 3-D Printers and Specimens Applied in the Lab

\begin{tabular}{|l|l|l|l|l|l|}
\hline \multirow{2}{*}{ Printer } & \multicolumn{3}{|c|}{ Campus B } & \multicolumn{2}{c|}{ Campus A } \\
\cline { 2 - 6 } & FlashForge & $\begin{array}{l}\text { Lulzbot } \\
\text { Mini }\end{array}$ & $\begin{array}{l}\text { MBot } \\
\text { Cube }\end{array}$ & Lulzbot TAZ & MBot Replicator 2X \\
\hline $\begin{array}{l}\text { Polymer } \\
\text { (number) }\end{array}$ & HIPS (5) & ABS (6) & PLA (6) & HIPS (10) & ABS (15) \\
\cline { 2 - 6 } & & & & PLA(10) & \\
\hline
\end{tabular}


Table 4 - Filament Specification for 3-D Printing

\begin{tabular}{|l|l|l|}
\hline Polymer & Density $\left(\mathrm{g} / \mathrm{cm}^{3}\right)$ & Extrusion Temperature $\left({ }^{\circ} \mathrm{C}\right)$ \\
\hline ABS & 1.07 & $230-240$ \\
\hline HIPS & -- & $220-230$ \\
\hline PLA & 1.25 & $205 \pm 15$ \\
\hline
\end{tabular}

The CNC-machining method was the process of cutting polymer sheets. ABS, PS, and ABS sheets were purchased online as polymer sheet, cut to 1 foot $(305 \mathrm{~mm})$ squares. Students used the $\mathrm{CNC}$ machine to cut approximately 12 samples from each sheet with the help of the lab technician. The specimen dimensions are shown in Table 1. Ten of the twelve cut specimens of each material were selected for tensile testing. The parts were smooth and flat, excepting some chips left at the edges which could easily be filed away. See table 5 for polymer sheets properties provided by the manufacturer.

Table 5 - Cut-to-size Sheet Specifications for CNC Machining Specimens ${ }^{4}$

\begin{tabular}{|l|l|l|l|}
\hline Polymer & Tensile Strength $(\mathrm{psi})$ & Impact Strength $(\mathrm{ft}-\mathrm{lb} / \mathrm{in})$ & Mold Temperature $\left({ }^{\circ} \mathrm{F}\right)$ \\
\hline ABS & 5100 & 5.20 & 160 \\
\hline LDPE & 3100 & -- & 120 \\
\hline PS & 2500 & 2.20 & 155 \\
\hline
\end{tabular}

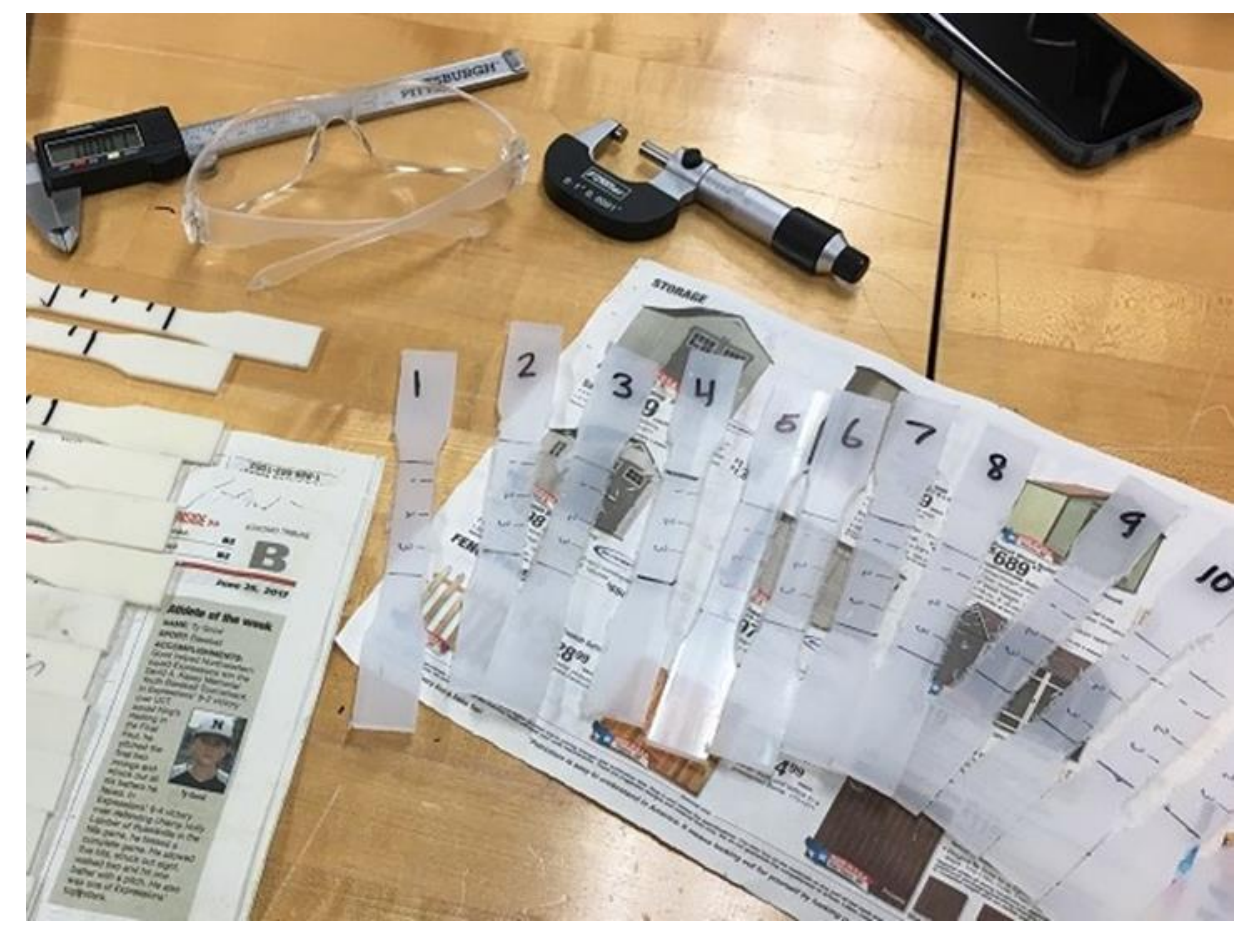

Figure 3 Clear cut-to-size LDPE specimens with measuring instruments and visible cross sectional area measurement locations 1,2 , and 3

Measurement and Testing 
Dimensional measurements were obtained before and after tensile testing. Although these freshly-made specimens' geometry should match the specifications in table 1, there were some small differences. Among the three manufacturing processes, the 3-D printed samples came closest to the standard dimensions while the injection molded parts varied the most. Figure 3 shows the measured LDPE specimens and dimensional measuring instruments (digital calipers, micrometer calipers, and a 1 -ft steel rule).

The detailed measuring and testing procedure was:

a. Measure the total length of each specimen.

b. Using a permanent marker, mark the two-inch gauge length on the narrow middle portion.

c. Select three locations within the gauge length and mark them for cross-sectional area dimensions. Make sure the three points are not too close to each other (see figure 3).

d. Use the micrometer calipers to measure the thickness at each of the three locations.

e. Use digital calipers to measure the width at each of the three locations.
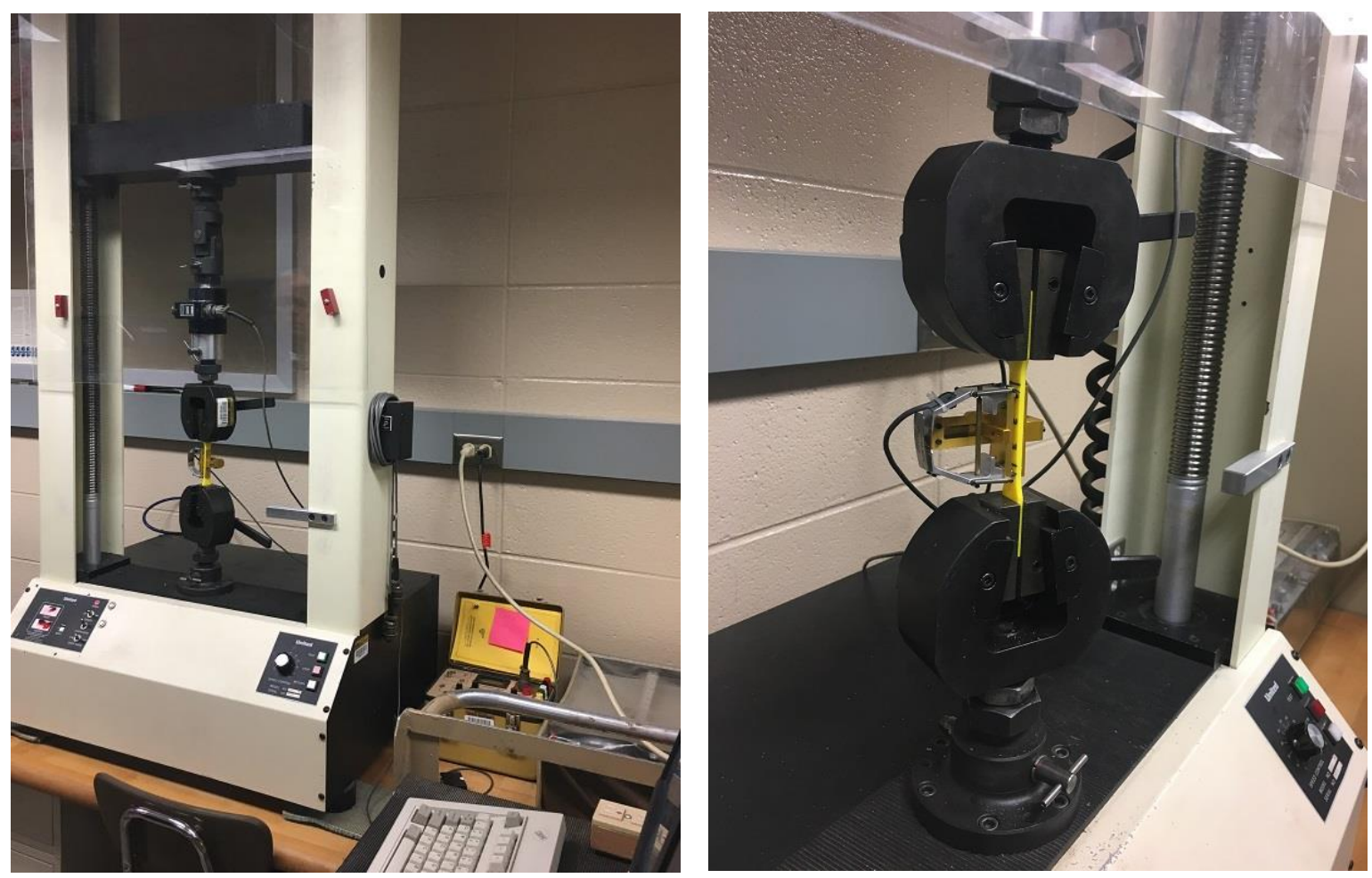

Figure 4 (left) Tensile test machine and (right) specimen in the grips with extensometer

f. Load the specimen into grips and place extensometer on it (shown in figure 4).

g. Run the test (load cell detects the pulling force and extensometer measures deformation).

h. Put the two broken parts together and measure the length between gauge marks after the test.

i. Measure the gauge length again.

j. Measure the width and thickness at the three marked locations again.

Working from the computer-acquired load and deformation data, student identified and recorded several important mechanical properties such as yield strength, ultimate tensile strength, and 
fracture stress. The engineering stress-strain curve of each test was also plotted from the results, making Young's modulus another property to consider. Students were also expected to generate the actual stress-strain curves using the cross-sectional dimensions of the specimen obtained at three locations within the gauge length in the original design of this lab project to determine the effect of variations in cross-section on strength (see steps $c$, $d$, and $j$ ). However, for the first run of the project, the students could not complete this portion due to the tight schedule at the end of the semester.

\section{Results}

Table 6 shows the difference of ABS tensile testing results among three processing methods. All the yield strength values were determined at a $0.2 \%$ offset. There is no consistent, significant difference between 3D-printed and cut-to-size CNC machined specimens' properties. However, the injection molded specimens have relative high strength and stiffness values when compared to ABS specimens from the other processes. In table 7, the 3D-printing material was HIPS, while the other two processes used PS. The differences in length change and fracture strength (and the corresponding toughness) were significant. However, the differences in yield strength and tensile strength were minimal. LDPE's testing results comparison appears in Table 8. (No LDPE specimen was made by the 3D printing process). Table 8 shows that cut-to-size machined LDPE parts were both stiffer and stronger than those that were injection molded. Beginning with table 9, properties of 3-D printed specimens of one material made by different printers are listed. The results between two printers were relatively similar, indicating the process has more effect on these properties than the processing equipment.

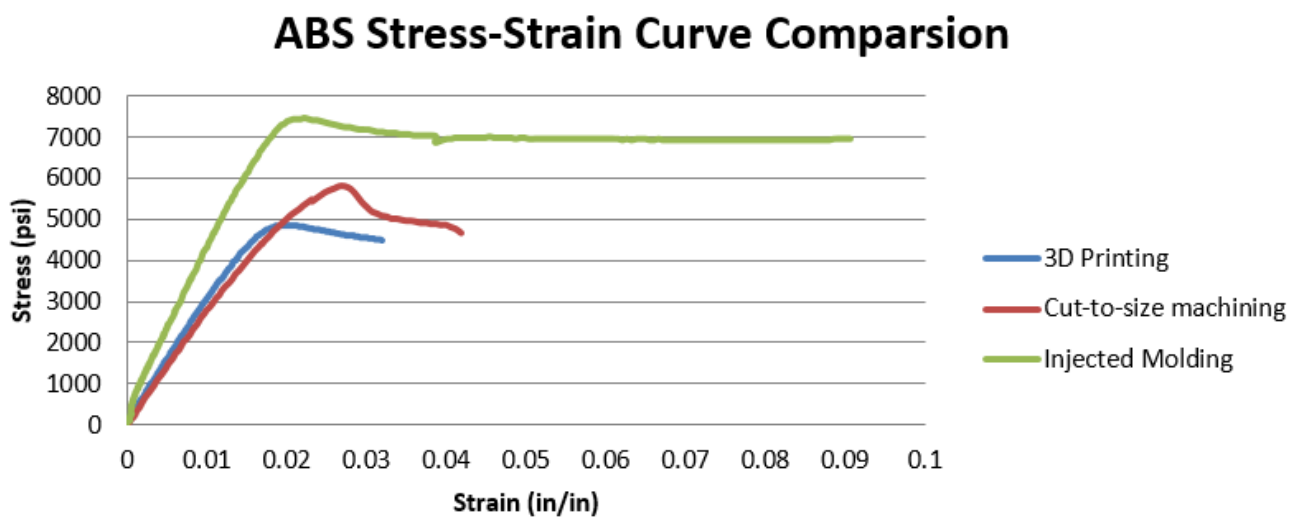

Figure 5 ABS stress-strain curves of three manufacturing processes

Published property data for the thermoplastics shown in table 7-9 only appear by range due to the many proprietary variations that are on the market ${ }^{5}$. For example, cut-to-size machined parts were listed as polymer sheet and the injection molded material was extruded as part of its pellet-forming process. The results of the students' work on this laboratory project do correlate with published data. For this portion of the study, students calculated strain based on the average cross-sectional area as determined from three measurements of width and thickness. 
Table 6 - ABS Specimens' Mechanical Properties Comparison by Process

\begin{tabular}{|l|l|l|l|l|l|}
\hline Process & $\begin{array}{l}\text { Gauge } \\
\text { Length } \\
\text { Change (in) }\end{array}$ & $\begin{array}{l}\text { Yield } \\
\text { Strength } \\
(\mathrm{psi})\end{array}$ & $\begin{array}{l}\text { Tensile } \\
\text { Strength } \\
(\mathrm{psi})\end{array}$ & $\begin{array}{l}\text { Fracture } \\
\text { Strength } \\
(\mathrm{psi})\end{array}$ & $\begin{array}{l}\text { Young's } \\
\text { Modulus } \\
(\mathrm{psi})\end{array}$ \\
\hline 3D Printing & 0.0373 & 3863 & 4853 & 4491 & 301234 \\
\hline CNC Machining & 0.0469 & 3956.5 & 5807 & 4364 & 271287 \\
\hline Injection Molding & 0.0573 & 7120 & 7432 & 6951 & 396812 \\
\hline
\end{tabular}

Table 7 - PS Specimens' Mechanical Properties Comparison by Process

\begin{tabular}{|l|l|l|l|l|l|}
\hline Process & $\begin{array}{l}\text { Gauge } \\
\text { Length } \\
\text { Change (in) }\end{array}$ & $\begin{array}{l}\text { Yield } \\
\text { Strength } \\
(\mathrm{psi})\end{array}$ & $\begin{array}{l}\text { Tensile } \\
\text { Strength } \\
(\mathrm{psi})\end{array}$ & $\begin{array}{l}\text { Fracture } \\
\text { Strength } \\
(\mathrm{psi})\end{array}$ & $\begin{array}{l}\text { Young's } \\
\text { Modulus } \\
(\mathrm{psi})\end{array}$ \\
\hline 3D Printing & 0.0879 & 2774 & 2815 & 1203 & 233916 \\
\hline CNC Machining & 0.0289 & 2678 & 2753 & 2248 & 239671 \\
\hline Injection Molding & 0.1150 & 3706 & 3934 & 3933 & 457278 \\
\hline
\end{tabular}

Table 8 - LDPE Specimens' Mechanical Properties Comparison by Process

\begin{tabular}{|l|l|l|l|l|l|}
\hline Process & $\begin{array}{l}\text { Gauge } \\
\text { Length } \\
\text { Change (in) }\end{array}$ & $\begin{array}{l}\text { Yield } \\
\text { Strength } \\
(\mathrm{psi})\end{array}$ & $\begin{array}{l}\text { Tensile } \\
\text { Strength } \\
(\mathrm{psi})\end{array}$ & $\begin{array}{l}\text { Fracture } \\
\text { Strength } \\
(\mathrm{psi})\end{array}$ & $\begin{array}{l}\text { Young's } \\
\text { Modulus } \\
(\mathrm{psi})\end{array}$ \\
\hline Injection Molding & 1.500 & 613 & 1157 & 1089 & 20204 \\
\hline CNC Machining & 1.330 & 803 & 1294 & 1205 & 29503 \\
\hline Difference \% & 11.3 & 23.7 & 10.6 & 9.62 & 31.5 \\
\hline
\end{tabular}

Table 9 - PLA Specimens' Mechanical Properties Comparison by Printer

\begin{tabular}{|l|l|l|l|l|l|}
\hline 3-D Printing & Campus Location & $\begin{array}{l}\text { Yield } \\
\text { Strength } \\
(\mathrm{psi})\end{array}$ & $\begin{array}{l}\text { Tensile } \\
\text { Strength } \\
(\mathrm{psi})\end{array}$ & $\begin{array}{l}\text { Fracture } \\
\text { Strength } \\
(\mathrm{psi})\end{array}$ & $\begin{array}{l}\text { Young's } \\
\text { Modulus } \\
\text { (psi) }\end{array}$ \\
\hline MBot Cube & West Lafayette & 6550 & 7045 & 7034 & 440562 \\
\hline Lulzbot TAZ & Kokomo & 5836 & 6983 & 6972.5 & 320413 \\
\hline Difference & $\%$ & 10.9 & 0.880 & 0.874 & 27.3 \\
\hline
\end{tabular}

Considering only 3D-printed ABS specimens at Campus B, initial measured dimensions for thickness and width at three locations within the gauge length were used to determine apparent ultimate strength, yield strength, and Young's modulus. For six specimens, these dimensions varied by a maximum of $0.076 \mathrm{~mm}$ ( $0.003 \mathrm{in})$. In most cases, the corresponding mechanical properties were the same if the values were reported to two significant figures. Table 10 shows the data and mechanical property results for ABS specimen 6, which includes one of the exceptions to the two-significant figures agreement. Increasing student awareness of the limitations on their experimental work, as well as the constraints on published property values is one goal of this enhanced materials testing project, as well as validating the need to question data, a key aspect of critical thinking. 
Table 10 - ABS Specimens’ Mechanical Properties Comparison by Location

\begin{tabular}{|c|l|l|l|l|l|}
\hline Location & Initial Width (in) & $\begin{array}{l}\text { Initial thicknes } \\
\text { (in) }\end{array}$ & $\begin{array}{l}\text { Yield } \\
\text { Strength } \\
(\mathrm{psi})\end{array}$ & $\begin{array}{l}\text { Tensile } \\
\text { Strength } \\
\text { (psi) }\end{array}$ & $\begin{array}{l}\text { Young's Modulus } \\
\text { (psi) }\end{array}$ \\
\hline 1 & 0.493 & 0.130 & 3158.06 & 3248.56 & 193696 \\
\hline 2 & 0.494 & 0.131 & 3127.61 & 3217.23 & 191829 \\
\hline 3 & 0.491 & 0.130 & 3170.92 & 3261.79 & 194485 \\
\hline
\end{tabular}

\section{Discussion}

Participating campus A students were mainly sophomores taking the required strength of materials course. Through this project, their recognition of manufacturing sources of experimental variation was clearly exhibited through their expressed concerns about the effect of injection molding bubbles and inadvertently curved tensile specimen faces on mechanical stiffness and strength. These students successfully posed key questions about their manufactured specimens and the corresponding experimental results. And, they determined how to seek answers through analysis and to challenge standard testing assumptions when the assumptions might not be valid. Students' learning interest was increased through the step-by-step processing and testing procedures and the follow-up consideration of variability and its effects.

The Campus B freshman student came into this project seeking an initial undergraduate research experience. His nearly solo efforts made him aware of the necessity of explicit technical communication in working across locations, the potential variation in processing quality that may occur when several manufacturers make a single product type (in his case, 3D printed tensile specimens), and that analysis assumptions must be validated if results are to be accepted.

To give the faculty insight into the students' views of experimental methods and research after completing this project, Campus A students were surveyed. $89 \%$ of the students indicated they now understand that the manufacturing process can affect a material's mechanical strength and stiffness properties, and $89 \%$ agree that testing multiple identical specimens is appropriate for property determination, showing a positive effect of their enhanced laboratory project work. Experimental research did not capture the interest of a majority of the students, however. While $78 \%$ noted that working on a team-based research project produced a better experience than doing similar work individually, only $44 \%$ responded favorably to the item, "Experimental research intrigues me." The reasons for this low level of interest among the Campus A students have not been determined, and unfortunately, a pre-project survey was not conducted so the authors do not know if the project succeeded in piquing the research interests of any students with no previous interest. Speculation as to the causes for the low interest range from a potential student belief that research involves too much work or that materials testing simply was not the students' preference to the mundane possibility that perspectives were skewed by this project concluding late in the term when thoughts of the upcoming exams and subsequent break. The Campus B student went into the project with a favorable view of experimental research which is believed to be unchanged due to the student's continuation with the project. 


\section{Conclusions/summary:}

The initial offering of this tensile testing laboratory project met its goals of increasing student awareness of the variability in tensile properties based on manufacturing and enhancing their observation and understanding of experimental research. This project was coupled with a similar hardness testing effort to reinforce student recognition of process-based effects on material properties and provide more experimental research practice. The instructors plan to revisit the project design to ensure the scope of the work and the students' results with more guided analysis of the process-based variability in hopes that this slight increase in research focus will produce more intriguing results for future students. Pre and post project surveys will be conducted to better understand which student views of research and experimental work are influenced by the enhanced laboratory project.

By combining the manufacturing process and property testing experiences, this project showed the students the connection between manufacturing processes and the mechanical properties of the parts produced. Also, by replicating the work at two locations and sharing the data gathered, students see how two seemingly alike processes being performed different locations can produce different manufacturing results. The learning outcomes of this laboratory better prepare engineering technology students for their future careers.

\section{Acknowledgments:}

Contributions to this project by YYYYY, Campus A laboratory technician, and ZZZZ, Campus $\mathrm{B}$ undergraduate research student, were significant and very much appreciated.

\section{References}

1. Black, J.T., and Kohser, R. (2012). DeGarmo's Materials and Processes in Manufacturing, $11^{\text {th }}$ edition, John Wiliey \& Sons, Incorporated.

2. ASTM D638 - 14 Standard Test Method for Tensile Properties of Plastics

3. Balani, K. Physical, Thermal, and Mechanical Properties of Polymers. (2015). onlinelibrary.wiley.com/doi/10.1002/9781118950623.app1/pdf

4. McMaster-Carr Supply company, www.mcmaster.com/

5. www. Matweb.com

6. Sahin, S and Yayla, P. (2005). Effects of processing parameters on the mechanical properties of polypropylene random copolymer. Polymer Testing. 24 (1012-1021).

7. "Coloring methods and Effect of coloring on properties." Polyplastics Solution Platform for Engineering Plastics. www.polyplastics.com/en/product/safety/coloring/. Accessed 18 March, 2018.

\section{Appendix A: Polymer Suppliers, Equipment, and Property references}

1) 3-D printers applied in this project / location

i. Lulzbot TAZ 5 - Kokomo

ii. MakerBot Replicator 2X-Kokomo

iii. MakerBot Cube - West Lafayette

iv. Lulzbox Mini - West Lafayette

v. FlashForge Creator - West Lafayette 
2) Filament material supplier webpages

- Gold PLA: https://www.matterhackers.com/store/l/pro-series-gold-pla-filament$3.00 \mathrm{~mm} / \mathrm{sk} / \mathrm{MYW} 2 \mathrm{EHZX}$

- HIPS (yellow is no longer available; this appears to be without pigmented): https://www.matterhackers.com/store/1/hips-300mm-1kg/sk/MFAM5YE9

- Silver ABS page: https://www.matterhackers.com/store/1/175mm-abs-filamentsilver-1-kg/sk/M5A2YT7Z

3) Cut-to-size Plastic Sheeting https://www.tapplastics.com/product/plastics/cut_to_size_plastic

LDPE sheet: 12 " x 12 " x $1 / 8$ "

Polystyrene Sheet: 12 " x 24 " x $1 / 8$ "

ABS Sheet: $12 " x 12 " x 1 / 8 "$

4) The following references are generic properties for molded plastic:

ABS:

http://matweb.com/search/DataSheet.aspx?MatGUID=eb7a78f5948d481c9493a67f0d089

$\underline{646}$

LDPE:

http://matweb.com/search/DataSheet.aspx?MatGUID=557b96c10e0843dbb1e830ceedeb $\underline{35 \mathrm{~b} 0}$

Polystyrene:

http://matweb.com/search/DataSheet.aspx?MatGUID=df6b1ef50ce84e7995bdd1f6fd1b0 $\underline{4 c 9}$

5) The following links show strength of generic filament materials: https://eu.makerbot.com/fileadmin/Inhalte/Support/Datenblatt/MakerBot_R_PLA_and_ ABS_Strength_Data.pdf https://3dprint.com/42417/3d-printing-material-strengths/ https://www.lifewire.com/3d-printed-material-strength-2230 http://www.sciencedirect.com/science/article/pii/S2214860416300859 


\section{Appendix B: Project survey items}

(Likert scale, 5 choices from strongly agree to strongly disagree).

1. I now understand the manufacturing process may affect the hardness of a material.

2. I now understand the manufacturing process may affect the tensile strength and stiffness of a material.

3. The ASTM requirement for testing multiple identical specimens is appropriate when determining material properties.

4. Establishing consistent test locations across specimens is important.

5. Specimen quality affects material properties.

6. Experimental research intrigues me.

7. Working with other students on a team improved my research project experience (when compared to doing an individual research project).

8. Comments 